\title{
A Belgian consensus protocol for autologous hematopoietic stem cell transplantation in multiple sclerosis
}

\author{
Guy Laureys ${ }^{1}\left[\right.$ [ $\cdot$ Barbara Willekens ${ }^{2} \cdot$ Ludo Vanopdenbosch $^{3} \cdot$ Olivier Deryck $^{3} \cdot$ Dominik Selleslag $^{4}$. \\ Miguel D'Haeseleer ${ }^{5}$. Ann De Becker ${ }^{6}$. Bénédicte Dubois ${ }^{7}$. Daan Dierickx ${ }^{8} \cdot$ Gaetano Perrotta $^{9}$. \\ Virginie De Wilde ${ }^{10}$. Vincent van Pesch ${ }^{11}$. Nicole Straetmans ${ }^{12}$. Dominique Dive ${ }^{13} \cdot$ Yves Beguin $^{14}$. \\ Bart Van Wijmeersch ${ }^{15} \cdot$ Koen Theunissen $^{16} \cdot$ Tessa Kerre $^{17} \cdot$ Ann Van de Velde $^{18}$
}

Received: 17 November 2017 / Accepted: 26 February 2018

(c) Belgian Neurological Society 2018

\begin{abstract}
Multiple sclerosis is considered to be an immune mediated inflammatory disorder of the central nervous system. It mainly affects young, socioeconomic active patients. Although our armamentarium for this disease has significantly evolved in recent years some patients remain refractory to conventional therapies. In these cases, autologous hematopoietic stem cell transplantation can be considered as a therapeutic option. Decreasing morbidity, mortality, and increasing patient awareness have led to rising inquiry by our patients about this treatment option. With the aim of a standardized protocol and data registration, a Belgian working party on stem cell therapy in multiple sclerosis was established. In this paper, we report the consensus protocol of this working party on autologous hematopoietic stem cell transplantation in multiple sclerosis.
\end{abstract}

Keywords Multiple sclerosis · Autologous hematopoietic stem cell transplantation · Consensus Paper

Electronic supplementary material The online version of this article (https://doi.org/10.1007/s13760-018-0905-0) contains supplementary material, which is available to authorized users.

Guy Laureys

laureysg@hotmail.com

1 Department of Neurology, Ghent University Hospital, De Pintelaan 185, 9000 Ghent, Belgium

2 Department of Neurology, Antwerp University Hospital, Wilrijkstraat 10, 2650 Edegem, Belgium

3 Department of Neurology, AZ Sint Jan Brugge Oostende, Ruddershove 10, 8000 Brugge, Belgium

4 Department of Hematology, AZ Sint Jan Brugge Oostende, Ruddershove 10, 8000 Brugge, Belgium

5 Department of Neurology, Center for Neurosciences, Universitair Ziekenhuis Brussel, Vrije Universiteit Brussel, Brussels, Belgium

6 Department of Hematology, UZ Brussel, Laarbeeklaan 101, 1090 Brussels, Belgium

7 Department of Neurology, UZ Leuven, Herestraat 49, 3000 Louvain, Belgium

8 Department of Hematology, UZ Leuven, Herestraat 49, 3000 Louvain, Belgium

9 Unité de neuroimmunologie, Service de Neurologie, Hôpital Erasme-Université Libre de Bruxelles, Brussels, Belgium
10 Department of Hematology, Hôpital Erasme-Université Libre de Bruxelles, Brussels, Belgium

11 Department of Neurology, Cliniques universitaires Saint-Luc, Université Catholique de Louvain, Avenue Hippocrate 10, 1200 Brussels, Belgium

12 Department of Hematology, Cliniques universitaires Saint-Luc, Université Catholique de Louvain, Avenue Hippocrate 10, 1200 Brussels, Belgium

13 Department of Neurology, University Hospital of Liege, Rue Grandfosse 31-33, 4130 Esneux, Belgium

14 Department of Hematology, University of Liège, CHU Sart Tilman, 4000 Liège, Belgium

15 Biomedical Institute, Hasselt University and Rehabilitation and MS-Centre Overpelt, Agoralaan Gebouw A, 3590 Diepenbeek, Belgium

16 Department of Hematology, Jessa Ziekenhuis, Stadsomvaart 11, 3500 Hasselt, Belgium

17 Department of Hematology, Ghent University Hospital, De Pintelaan 185, 9000 Ghent, Belgium

18 Department of Hematology, Antwerp University Hospital, Wilrijkstraat 10, 2650 Edegem, Belgium 


\section{Background}

Multiple sclerosis (MS) is an immune mediated inflammatory disorder of the central nervous system, with a prevalence of 83 per 100,000 in Europe [1] corresponding with an estimated prevalence of 88 per 100,000 in a 1991 survey in Flanders, Belgium [2]. Classically the disease has been divided into a relapsing-remitting variant (RRMS) and progressive forms: secondary progressive (SPMS) if preceded by initial RRMS or primary progressive (PPMS) if progressive from disease onset. The immunopathology of relapsing forms is thought to be driven by a peripheral and adaptive autoimmune response, whereas progressive forms are the consequence of a diffuse innate immune response within the CNS and neurodegenerative mechanisms triggered by uncontrolled chronic neuroinflammation [3]. This immunopathological distinction is important since autologous hematopoietic stem cell transplantation (AHSCT) mainly targets the peripheral adaptive inflammatory arm of the disease and thus should only be considered in aggressive, treatment-refractory RRMS, and progressive MS with substantial signs of ongoing inflammatory disease. Such an aggressive disease course has been estimated to occur in $4-14 \%$ of patients depending on the definition used [4-6]. Although a substantial increase in our therapeutic armamentarium has changed our treatment of MS in a drastic way [7] optimal disease control remains elusive for many of these patients. Originally supported by animal models and serendipitous clinical reports, AHSCT has been evolving since 1996 as a potential treatment for patients with severe autoimmune disease (ADs) refractory to conventional treatments. With this emerging clinical practice, the European Group for Blood and Marrow Transplantation (EBMT) Autoimmune Disease Working Party (ADWP) was created to perform multicenter retrospective studies and organize randomized controlled trials in MS and other ADs [8, 9]. In 2012 Mancardi and colleagues reported the results of the Italian multicenter retrospective study including 74 patients treated with AHSCT with a BEAM/ATG conditioning regimen showing a sustained effect in suppressing disease progression (66\% had stable or improved EDSS scores after 5 years). Patients in the relapsing-remitting phase of the disease were noted to benefit specifically [10]. In 2015 Burt and colleagues reported on 145 (123 relapsing-remitting MS, 28 secondary progressive) patients who underwent nonmyeloablative (cyclophosphamide and ATG in 129, alemtuzumab and cyclophosphamide in 22 patients) and unmanipulated hematopoietic stem cell transplantation [11]. This study showed similar results as the Mancardi cohort with significant improvement in disability (decrease in EDSS score of $\geq 1.0$ ) in 41 patients $(50 \%)$ at 2 years and in 23 patients
(64\%) at 4 years. Four-year relapse-free survival was $80 \%$ and progression-free survival was $87 \%$. Moreover, the AHSCT-related mortality rate was $0 \%$ in this retrospective cohort. Currently, a randomized phase 3 study, called the MS International Stem Cell Transplant (MIST) trial, is underway to investigate AHSCT with a low-intensity conditioning regimen versus standard treatment in patients with RRMS. Results are expected to be available in the following year. Recently, Nash and colleagues published their 5-year follow-up results of the Hematopoietic Cell Transplantation for relapsing-remitting multiple sclerosis (HALT-MS) trial, a prospective multicenter, single-arm, phase 2 clinical trial of high-dose immunosuppressive therapy ("HDIT", in this case the BEAM protocol with CD34 + selection) and AHSCT for patients with RRMS. This study provided Class IV evidence that participants with RRMS experienced sustained remissions $(91.3 \%$ of patients had stable or better EDSS scores with a mean follow-up of 62 months) with toxicities as expected from HDIT/AHSCT and a treatment-related mortality rate of $0 \%$ [12]. The most stringent criteria of treatment effectiveness, termed "No Evidence of Disease Activity" (NEDA: defined as absence of clinical and radiological disease activity [13]), are only attained in a fraction of conventionally (mainly interferons and copolymer) treated patients (7.9\%) as witnessed by the cohort of Rotstein and colleagues [14]. Compared to these "conventional" treatment cohorts, AHSCT demonstrates much higher proportions of NEDA at 2 years (ranging in studies from 78 to $83 \%$ ) and at 5 years (60-68\%) [15]. These data seem even more impressive considering that MS patients who underwent AHSCT have much more aggressive disease if compared to clinical trial or cohort populations. Although safety has improved mortality rates for AHSCT in MS are still around 1-2\% [16] and thus should only be considered in patients with highly active inflammatory disease [17]. A recent meta-analysis [18] summarizes the evidence for AHSCT in MS as derived from pooled data on AHSCT in 764 transplant patients from 15 studies (in any form of MS) over a 21-year period (1995-2016). The meta-analysis demonstrated a transplant-related mortality of $2.1 \%$, estimates being higher in trials conducted at earlier dates, for patients with a higher baseline EDSS, and for studies with a lower proportion of RRMS patients. The pooled rate of disease progression at 2 years was $17.1 \%$, with $23.3 \%$ at 5 years. No evidence of disease activity was achieved in 83 and $67 \%$ of patients at 2 and 5 years, respectively. A recent review by the same authors [19] as the meta-analysis concluded that further trials are needed but stated that they "believe that enough evidence already exists to support the use of AHSCT for treatment of patients with aggressive RRMS and those with active RRMS in whom highpotency, approved, disease-modifying therapy has failed 
because of a lack of efficacy ... We advocate healthcare organizations in all other countries to consider introducing AHSCT as the standard of care for these indications, and to regularly reassess and update their guidelines on the basis of new evidence that could alter the indications".

\section{Methodology of consensus agreement}

Within the Belgian patient population awareness of AHSCT as a therapeutic option for MS has grown, necessitating clear national guidelines for centers to support correct patient counseling. Moreover, uniform guidelines promote qualitative treatment and research perspectives. In this context, a working party on hematopoietic stem cell transplantation in multiple sclerosis was formed. Members were recruited based on the presence of combined center expertise in AHSCT (clinical hematologists) and MS (neurologists). A questionnaire for neurologists and hematologists was conceived (see Supplementary Files 1 and 2) based on the 2012 guidelines of the EBMT for AHSCT in severe autoimmune diseases [20]. All hematologists (9/9) and neurologists (9/9) filled out the questionnaire followed by separate hematologist and neurologist's meetings to reach consensus on the place and modalities of AHSCT in the treatment algorithm of MS.

\section{Aims and goals of the Belgian MS-AHSCT working party}

After discussion, the following aims and goals of the working party were defined:

1. Establish a clear protocol and guidelines for AHSCT in the setting of adult Belgian MS patients.

2. Support and promote research in the field of AHSCT and other experimental (stem)-cell strategies (mesenchymal/ other, etc.) in MS.

3. Promote continued education in the field of AHSCT for MS from a hematological and neurological perspective.

4. Organize a Belgian registry for AHSCT in MS.

\section{AHSCT in MS: common decisions from neurologists and hematologists}

Since correct patient counseling and data registration demand concerted evaluation and follow-up by hematologists and neurologists the following decisions were made by the members of the working party:
Multidisciplinary evaluation by the neurologist and hematologist should take place at initial evaluation, day 100 after transplant, and yearly thereafter. This was agreed upon by all the participating members. Time points of follow-up reporting are according to EBMT registration data (https://www.ebmt.org/Contents/Data-Management /Registrystructure/MED-ABdatacollectionforms/Docum ents/13MS.pdf). At these moments neurologists and hematologists should exchange all necessary clinical data for optimal patient care and qualitative data registration as defined in paragraph 4.1. The way neurologists and hematologists discuss the patient consult can be defined based on the practicalities of the specific centers. More regular follow-up on an individual basis can be planned as needed according to good clinical practice.

Approval of the AHSCT protocol should be obtained from local ethics committees. Treatment protocols do not necessitate the approval of the ethics committee [21].

As recommended in the EBMT 2012 guidelines continual education within the applicable auto-immune domain (e.g. multiple sclerosis) is advised [20]. This was agreed upon by all members as a mandatory element of good clinical practice. During a yearly reunion research perspectives, protocol updates and AHSCT requests will also be reviewed.

Since the EBMT proposes AHSCT in autoimmune diseases as "clinical opinion (CO)" written informed consent should be obtained from all patients. Informed consent for AHSCT is already standard of care in all JACIE accredited stem cell transplant centers (http://www.jacie.org/stand ards/6th-edition-2015).

Biobanking is EBMT recommendation [20] and possible in all 9 centers. Funding should be sought for systematic prospective biobanking.

The need for an external reviewing committee reviewing the file "on paper" after the initial multidisciplinary evaluation in the treating center was discussed. Since inclusion criteria are amenable for discussion, an external reviewing committee could be installed to provide uniform advice for patients and referring neurologists. After discussion, it was concluded that all "clear cut cases" meeting all neurological and hematological eligibility criteria as stated in the consensus protocol should not need compulsory external reviewing. When in doubt or when deviation of standard criteria is deemed to be justified this should be discussed with the other members of the working party. Therefore, the patient file should be submitted to two neurologists and two hematologist members of the working party (external to the requesting center) and agreement on the indication for AHSCT should be agreed on by the four questioned physicians. During the yearly joint reunion, the protocol will be adjusted to current state of the art knowledge. 
Since criteria, effectiveness, and risks of AHSCT are still a matter of debate, inclusion of patients in a phase 3 study should be considered if available.

\section{AHSCT in MS: registry and indications from a neurological perspective}

\section{Registry of AHSCT data from a neurological perspective}

Patient data on disease characteristics before and after AHSCT will be documented in a registry. Thus, timelines and all examinations were designed to be according to standard of care and good clinical practice as defined by the working party, stated below. Data will be registered in the local database of the involved center (iMED, Edmus, etc.) and submissions of data in national (BELTRIMS) and international (MSBase [22]) registries are encouraged. Retrospective registration of disease course should be as detailed as possible for (at least) 2 years preceding the procedure. In order to optimize registration of the preceding disease course registration of patient data should be optimized in all Belgian (referral) centers. Follow-up as defined should be within the reference center. If a patient wants to change his follow-up center he should strongly be recommended to choose one of the other centers as defined in the working party. A reference center is defined as a center with a JACIE- accredited transplant unit and a neurologist with expertise in MS that have the necessary facilities to be compliant with the consensus protocol. For most centers of the working party the combined expertise is within the same hospital-facility, for the MS and revalidatie centrum Overpelt transplants will be performed at the Jessa-hospital Hasselt. Of course, other centers can join the working party to apply as a reference center.

After discussion imaging follow-up with Magnetic Resonance Imaging (MRI) of the Brain and Spinal Cord was defined as follows:

- Timepoints: 1 month preceding the procedure, afterwards on month $6,12,18,24$, and thereafter on a yearly basis.

- Minimal brain MRI sequences were defined as: 3D Flair with thin slices, $\mathrm{T} 1+-\mathrm{Gad}$, and 3D T1 consistent with MAGNIMS recommendations for follow-up scanning [23]. The sequences encompass required elements for later volumetric assays. Since concerns were voiced about the potential accumulation of gadolinium with repeated imaging [24], evolving knowledge on this potential long-term risk will be monitored and consequent adjustments to the protocol will be made according to new data.
- MRI of (at least the cervical) spinal cord will be performed the month preceding the procedure, after 1 year and thereafter with 2-yearly intervals. Sequences should include STIR or PD, T2, and T1 (with and without Gd) consistent with MAGNIMS recommendations [23].

- MRI follow-up should be performed on the same MRImachine as much as possible to avoid inter-scanner variation [25].

The clinical follow-up was defined as next:

- Follow-up of Expanded Disability Status Scale (EDSS) with full evaluation of the Functional Systems (FS) scores on the month preceding the procedure and at months 3, 6 and thereafter at 6-monthly intervals.

- Registration of 9-hole peg test, timed 25 Foot-walk, and symbol-digit modality test will be collected at the month preceding and thereafter yearly intervals. If time permits, the Brief International Cognitive Assessment for Multiple Sclerosis (BICAMS) is advised for cognitive followup.

- Relapse history will be documented with description of symptomatology, anatomical location, need for steroid treatment (oral, intravenous, dosage, duration), duration of relapse, impact on EDSS with FS components should be documented during the relapse episode as well as the degree of recovery afterwards.

- Registration of side effects and complications. The potential relation to AHSCT should be documented.

- Treatment: Registration of all medications including Disease Modifying Treatments (DMT's) and symptomatic therapies.

\section{Criteria for AHSCT in RRMS patients}

The following inclusion criteria were defined:

1. Age between 18 and 60 years.

2. MS diagnosis confirmed by McDonald criteria (2010) [26].

3. Maximum EDSS of 6.5 (Exception for higher EDSS in cases of "fulminant" MS or important EDSS increase induced by a relapse in the past year with potential for recovery).

4. Disease duration $\leq 15$ years from MS diagnosis.

5. Treatment failure after at least one highly effective treatment $(2$ courses of alemtuzumab or at least 6 months of treatment with mitoxantrone, cyclophosphamide, natalizumab, rituximab, ocrelizumab). 
6. Treatment failure is defined as the presence of the following criteria after at least 6 months of "highly effective therapy (see point 5)":

- A documented clinical relapse.

AND

- MRI activity (brain or spinal cord imaging) defined as compared to a baseline scan (the time point of the baseline scan is not strictly defined as it can depend on the specific DMT) after initiation of highly effective treatment:

$-\geq 1 \mathrm{Gad}+$ lesion and/or:

$-\quad \geq 2$ new T2 lesions.

The following exclusion criteria were defined:

1. Patients not reliable to understand the risks and benefits of the procedure or unable to give written informed consent.

2. Previous treatment with AHSCT.

3. Contra-indication or inability to undergo MRI scans.

4. Recent suicide attempt or serious uncontrolled depression.

5. Hematological exclusion criteria as described in "Recommendations for exclusion criteria for HSCT".

\section{Criteria for progressive patients with active disease}

Although initially separate criteria were proposed for patients with primary and secondary progressive disease the working party decided that the 2013 revisions defining the clinical course of multiple sclerosis should be used [27]. In these criteria, progressive disease [whether from onset (primary progressive) or after an initial relapsing course (secondary progressive)] is divided into four groups depending on disease (in)activity (clinical relapses, gadolinium-enhancing activity, or new or unequivocally enlarging T2 lesions during the assessment period) and presence or absence of disease progression (defined as clinical evidence of disease progression, independent of relapses, over a given period of time in patients who have a progressive disease course). The working party members agreed that AHSCT should only be considered in progressive patients (whether primary or secondary) with important inflammatory disease activity. In this mindset, the following criteria for active progressive disease (with or without progression) were defined.
The following inclusion criteria were defined:

1. Age between 18 and 60 years.

2. MS diagnosis confirmed by McDonald criteria (2010). For primary progressive patient's presence of OCB is deemed necessary.

3. Maximum EDSS of 6.5 (Exception for higher EDSS in cases of "fulminant" MS or important EDSS increase induced by a relapse in the past year with potential for recovery).

4. Documented ongoing Progression since $<5$ years. Total disease duration of less than 15 years.

5. Disease activity defined by the following criteria in the last 12 months:

- A documented clinical relapse. AND

- MRI activity (brain or spinal cord imaging) in the last 12 months defined as compared to a previous scan in the last 2 years:

$-\geq 1 \mathrm{Gd}+$ lesion and/or:

$-\quad \geq 2$ new T2 lesions.

6. Since ocrelizumab is available in compassionate use for primary progressive patients these patients should only be considered in case of ocrelizumab treatment failure. Defined as the presence of the following after at least 6 months of treatment:

- A documented clinical relapse. AND

- MRI activity (brain or spinal cord imaging) in the last 12 months defined as compared to a baseline scan (for the specific case of ocrelizumab the baseline scan should take place at least after 8 weeks of treatment (based on ocrelizumab's documented activity MRI activity at week 8 [28]):

$-\quad \geq 1 \mathrm{Gd}+$ lesion and/or:

$-\geq 2$ new T2 lesions.

For exclusion criteria see "Criteria for AHSCT in RRMS patients". 


\section{AHSCT in MS: criteria, procedure and registration from a hematological perspective}

\section{Registry of AHSCT data from a hematological perspective}

All participating hematologists agreed with mandatory EBMT registration (MED-AB Data Collection Forms) at designated time points (day 0, 100, and yearly thereafter). As stated previously these time points correspond with multidisciplinary (neurologist-hematologist) patient follow-up and case discussion. Cases of failure from mobilization should be reported to the EBMT or other registry. There is a need for lifelong follow up for secondary malignancies.

\section{Recommendations for PBSC mobilization}

- Before mobilization and HSCT, consideration should be given to chemotherapy-induced infertility (semen, oocyte or embryo cryopreservation as appropriate), risk of induction of premature menopause, and ultimate need for hormone replacement therapy, where appropriate. Pregnancy should be excluded within 7 days of administering mobilization or conditioning chemotherapy.

- Autologous stem cells may be derived from PB or BM. Mobilized PBSCs are preferred based on ease of procurement and better engraftment characteristics.

- Mobilization procedures and stem cell processing should be performed in JACIE accredited collection centers.

- Priming chemotherapy is recommended to enhance mobilization while maintaining disease control and to prevent potential flare, which may be a consequence of G-CSF alone.

- The recommended mobilization regimen is $\mathrm{CY}$ at $2 \mathrm{~g} / \mathrm{m}^{2}$ with uromitexan (Mesna) protection and cautious hyperhydration followed by granulocyte-colony stimulating factor (G-CSF) 5-10 $\mu \mathrm{g} / \mathrm{kg}$ (level II).

- Before using CY for hematopoietic stem cell mobilization in MS: electrocardiogram, and cardiac ultrasound evaluation in view of potentially fatal cardiac toxicity.

- A minimum dose of between 3 and $5 \times 10^{6} \mathrm{CD} 34+$ cells/ $\mathrm{kg}$ should be collected.

- Back-up harvest is not recommended as a standard of care, especially since graft manipulation will not be undertaken.

- When CY-primed mobilization fails, a second attempt at PBSC mobilization or BM harvest should be considered following avoidance of immunosuppressive drugs, where possible. Despite the lack of evidence in patients with $\mathrm{AD}$, the use of plerixafor (Not reimbursed in Belgium, only reimbursed for lymphoma and myeloma, if poor mobilization to be negotiated with pharmaceutical company) and G-CSF may be reasonable in poor mobilizers after weighing up the benefits and risks. Steroid cover should be considered to reduce risk of disease flare related to G-CSF.

\section{Recommendations for conditioning regimen}

Cyclophosphamide ( $200 \mathrm{mg} / \mathrm{kg}$ in 4 days $)+$ In vivo $\mathrm{T}$ cell depletion with ATG (Thymoglobuline ${ }^{\circledR}$ ) $0.5 \mathrm{mg} / \mathrm{kg}$ of thymoglobulin (administered intravenously) 5 days before stem cell infusion, $1.0 \mathrm{mg} / \mathrm{kg} 4$ days before, and $1.5 \mathrm{mg} / \mathrm{kg}$ on 3 , 2 , and 1 day before stem cell infusion [11]. With slow ATG administration concomitant steroids, antihistaminics, and antipyretics should be administrated as per local guidelines.

On day $0, \mathrm{CD} 34+$ hematopoietic progenitor cells will be thawed and infused.

Filgrastim $(5 \mu \mathrm{g} / \mathrm{kg} /$ day $)$ will be administered from day +5 until recovery of blood counts.

\section{Recommendations for graft manipulation}

There is no evidence to support ex vivo graft manipulation, although decisions can be made on an individual patient basis. CD34 selection is not reimbursed in Belgium, increases the risk of infection, increases the recovery time of peripheral blood cell counts and requires harvesting a higher number of cells and is thus not recommended.

\section{Recommendations for exclusion criteria for HSCT}

- Pre-HSCT evaluation of heart, lung, kidney, and gastrointestinal function is critically important. Patients with advanced cardiac disease (left ventricular ejection fraction $<40 \%$, uncontrolled ventricular arrhythmias, pericardial effusions), renal insufficiency $(<30 \mathrm{~mL} /$ $\mathrm{min} / \mathrm{m}^{2}$ ), respiratory disease (clinical/subclinical ventilatory impairment due to respiratory muscle involvement in MS) or active gastrointestinal bleeding should be excluded.

- Any uncontrolled acute or chronic infection, including HIV, human T-lymphotropic virus type 1 and 2, hepatitis B surface antigen positivity, and hepatitis C PCR positivity, should be considered as a contraindication.

- Pregnancy should always be excluded within 7 days of administering mobilization chemotherapy or HSCT with a blood-based beta-human chorionic gonadotrophin assay. 


\section{Recommendations for infection prophylaxis and treatment}

\section{Prophylaxis}

- Pre-transplant workup should include screening for CMV, HSV, VZV, EBV, HIV, human T-lymphotropic virus type 1 and 2, hepatitis viruses and toxoplasmosis in all patients, with other infection screening appropriate for geographical location.

- CMV Ab-positive patients receiving ATG or other serotherapy, or receiving manipulated autografts, are recommended to undergo CMV PCR or antigenaemia screening for the first 100 days post-transplant.

- EBV Ab-positive patients receiving ATG or other serotherapy, or receiving manipulated autografts, are recommended to undergo EBV PCR screening for the first 100 days post-transplant, with active surveillance for post-transplant lymphoproliferative disease according to local practice.

- All patients should be accommodated in rooms, with appropriate clean air facilities (for example, laminar flow or HEPA) according to JACIE accreditation standards during BM aplasia/severe neutropenia. Since the graft is $\mathrm{T}$ cell depleted these standards are according to allogeneic transplantation.

- All patients should receive broad-spectrum antibacterial (for example, quinolones) and anti-fungal prophylaxis (for example, azoles) during aplasia period and herpes prophylaxis (acyclovir) during at least 100 days post-transplant.

- All patients should receive prophylaxis against Pneumocystis jiroveci (PCJ) (for example, oral co-trimoxazole (TMP/SMX) 3 times weekly as tolerated or, if not tolerated, alternatives, such as monthly nebulized pentamidine, dapsone or atovaquone) for at least 100 days post-transplant.

- All patients positive for anti-toxoplasma antibodies should receive oral co-trimoxazole (TMP/SMX) daily until day -1 , then after reconstitution of blood counts three times weekly for at least 100 days post-transplant.

- Consideration should be made to risk of reactivation of tuberculosis, with prophylaxis through the period of immune suppression where appropriate (level II).

- In carefully selected cases after weighing up the benefits, risks, and costs, and only when repeated serious infections intravenous immunoglobulines replacement therapy may be considered.

\section{Pre-emptive therapy}

May differ according to local center guidelines. If CD4 counts are used the following apply:

- Monitoring of CD4 counts once monthly until recovery to $>400 / \mu \mathrm{L}$

- Prophylaxis for PCJ and VZV until CD4 counts $>400 /$ $\mu \mathrm{L}$

- Monitoring for CMV and EBV until CD4 counts >400/ $\mu \mathrm{L}$

- CMV reactivation (diagnosed by PCR or Ag) should be treated with intravenous ganciclovir (standard), oral valganciclovir (medical need though Belgian Hematology Society) or intravenous foscarnet according to centre policy and protocols.

\section{Therapy of fever and proven infections}

- Treatment of fever and established infection should follow center policy and protocols since they may result in neurological deterioration in patients with MS.

- When a patient presents a new, high, and well-tolerated fever at the time of neutrophil recovery, engraftment syndrome should be considered along with infective causes.

\section{Recommendations for blood transfusion}

- Platelet and erythrocyte transfusions should be administered according to center policy and protocols. Blood products should be irradiated.

\section{Conclusion}

AHSCT is a therapeutic option for treatment refractory MS. A Belgian Working party has been formed to standardize the indication, procedural measures, and follow-up in patients treated with AHSCT as described in this paper. Data will be collected in a prospective way to evaluate the results of this treatment option in the rapidly evolving treatment algorithm for MS.

\section{Compliance with ethical standards}

Ethical approval This article does not contain any studies with human participants or animals performed by any of the authors. 
Conflict of interest DS received travel grants, speaker's fees and advisory board honoraria from Sanofi, producer of Thymoglobulin, the other authors declare no conflicts of interest concerning this paper.

Informed consent For this type of study, formal consent is not required.

\section{References}

1. Pugliatti M, Rosati G, Carton H, Riise T, Drulovic J, Vecsei L et al (2006) The epidemiology of multiple sclerosis in Europe. Eur J Neurol 13(7):700-722

2. van Ooteghem P, D'Hooghe MB, Vlietinck R, Carton H (1994) Prevalence of multiple sclerosis in Flanders, Belgium. Neuroepidemiology 13(5):220-225

3. Dendrou CA, Fugger L, Friese MA (2015) Immunopathology of multiple sclerosis. Nat Rev Immunol 15(9):545-558

4. Lublin FD, Reingold SC (1996) Defining the clinical course of multiple sclerosis: results of an international survey. National Multiple Sclerosis Society (USA) Advisory Committee on Clinical Trials of New Agents in Multiple Sclerosis. Neurology 46(4):907-911

5. Gholipour T, Healy B, Baruch NF, Weiner HL, Chitnis T (2011) Demographic and clinical characteristics of malignant multiple sclerosis. Neurology 76(23):1996-2001

6. Menon S, Shirani A, Zhao Y, Oger J, Traboulsee A, Freedman MS et al (2013) Characterising aggressive multiple sclerosis. J Neurol Neurosurg Psychiatry 84(11):1192-1198

7. Comi G, Radaelli M, Soelberg Sorensen P (2017) Evolving concepts in the treatment of relapsing multiple sclerosis. Lancet 389(10076):1347-1356

8. Fassas A, Passweg JR, Anagnostopoulos A, Kazis A, Kozak T, Havrdova E et al (2002) Hematopoietic stem cell transplantation for multiple sclerosis. A retrospective multicenter study. J Neurol 249(8):1088-1097

9. Mancardi GL, Sormani MP, Gualandi F, Saiz A, Carreras E, Merelli E et al (2015) Autologous hematopoietic stem cell transplantation in multiple sclerosis: a phase II trial. Neurology 84(10):981-988

10. Mancardi GL, Sormani MP, Di Gioia M, Vuolo L, Gualandi F, Amato MP et al (2012) Autologous haematopoietic stem cell transplantation with an intermediate intensity conditioning regimen in multiple sclerosis: the Italian multi-centre experience. Mult Scler 18(6):835-842

11. Burt RK, Balabanov R, Han X, Sharrack B, Morgan A, Quigley K et al (2015) Association of nonmyeloablative hematopoietic stem cell transplantation with neurological disability in patients with relapsing-remitting multiple sclerosis. JAMA 313(3):275-284

12. Nash RA, Hutton GJ, Racke MK, Popat U, Devine SM, Steinmiller KC et al (2017) High-dose immunosuppressive therapy and autologous HCT for relapsing-remitting MS. Neurology 88(9):842-852

13. Giovannoni G, Turner B, Gnanapavan S, Offiah C, Schmierer $\mathrm{K}$, Marta M (2015) Is it time to target no evident disease activity (NEDA) in multiple sclerosis? Mult Scler Relat Disord 4(4):329-333
14. Rotstein DL, Healy BC, Malik MT, Chitnis T, Weiner HL (2015) Evaluation of no evidence of disease activity in a 7-year longitudinal multiple sclerosis cohort. JAMA Neurol 72(2):152-158

15. Sormani MP, Muraro PA, Saccardi R, Mancardi G (2017) NEDA status in highly active MS can be more easily obtained with autologous hematopoietic stem cell transplantation than other drugs. Mult Scler 23(2):201-204

16. Curro D, Mancardi G (2016) Autologous hematopoietic stem cell transplantation in multiple sclerosis: 20 years of experience. Neurol Sci 37(6):857-865

17. Freedman MS, Rush CA (2016) Severe, highly active, or aggressive multiple sclerosis. Continuum (Minneap Minn) 22(3):761-784

18. Sormani MP, Muraro PA, Schiavetti I, Signori A, Laroni A, Saccardi R et al (2017) Autologous hematopoietic stem cell transplantation in multiple sclerosis: a meta-analysis. Neurology 88(22):2115-2122

19. Muraro PA, Martin R, Mancardi GL, Nicholas R, Sormani MP, Saccardi R (2017) Autologous haematopoietic stem cell transplantation for treatment of multiple sclerosis. Nat Rev Neurol 13(7):391-405

20. Snowden JA, Saccardi R, Allez M, Ardizzone S, Arnold R, Cervera $R$ et al (2012) Haematopoietic SCT in severe autoimmune diseases: updated guidelines of the European Group for Blood and Marrow Transplantation. Bone Marrow Transpl 47(6):770-790

21. Meulenbergs T, Vermylen J, Schotsmans PT (2005) The current state of clinical ethics and healthcare ethics committees in Belgium. J Med Ethics 31(6):318-321

22. Butzkueven H, Chapman J, Cristiano E, Grand'Maison F, Hoffmann M, Izquierdo G et al (2006) MSBase: an international, online registry and platform for collaborative outcomes research in multiple sclerosis. Mult Scler 12(6):769-774

23. Rovira A, Wattjes MP, Tintore M, Tur C, Yousry TA, Sormani MP et al (2015) Evidence-based guidelines: MAGNIMS consensus guidelines on the use of MRI in multiple sclerosis-clinical implementation in the diagnostic process. Nat Rev Neurol 11(8):471-482

24. Olchowy C, Cebulski K, Lasecki M, Chaber R, Olchowy A, Kalwak K et al (2017) The presence of the gadolinium-based contrast agent depositions in the brain and symptoms of gadolinium neurotoxicity-a systematic review. PLoS ONE 12(2):e0171704

25. Filippi M, van Waesberghe JH, Horsfield MA, Bressi S, Gasperini C, Yousry TA et al (1997) Interscanner variation in brain MRI lesion load measurements in MS: implications for clinical trials. Neurology 49(2):371-377

26. Polman CH, Reingold SC, Banwell B, Clanet M, Cohen JA, Filippi $M$ et al (2011) Diagnostic criteria for multiple sclerosis: 2010 revisions to the McDonald criteria. Ann Neurol 69(2):292-302

27. Lublin FD, Reingold SC, Cohen JA, Cutter GR, Sorensen PS, Thompson AJ et al (2014) Defining the clinical course of multiple sclerosis: the 2013 revisions. Neurology 83(3):278-286

28. Kappos L, Li D, Calabresi PA, O'Connor P, Bar-Or A, Barkhof F et al (2011) Ocrelizumab in relapsing-remitting multiple sclerosis: a phase 2, randomised, placebo-controlled, multicentre trial. Lancet 378(9805):1779-1787 